\title{
Use of organic amendments in table grape: effect on plant root system and soil quality indicators
}

\author{
M. Mercedes Martínez ${ }^{1,2 *}$, Rodrigo Ortega ${ }^{1,}$, Marc Janssens $^{2}$, Paola Fincheira ${ }^{1,3}$.
}

${ }^{1}$ Grupo de Investigación en Suelo, Planta, Agua y Ambiente (GISPA). Universidad Técnica Federico Santa María. Av. Santa Maria 6400, Vitacura- Santiago, Chile. Postal code: 7660251.

${ }^{2}$ TROPEN-Tropical Crops, Institute of Crop Science and Resource Conservation INRES Bonn Universität, Bonn Germany Auf dem Hügel 6, 53121 Bonn.

${ }^{3}$ Programa de Doctorado en Ciencias de Recursos Naturales, Universidad de La Frontera, Temuco, Chile.

*Corresponding author: mmmartinez@uni-bonn.de

\begin{abstract}
The application of different organic matter sources as soil amendments is a common practice to stimulate root development and soil quality to improve crop yield and quality. For evaluating the effect of organic amendments on soil quality indicators and agronomic variables in table grape (Vitis vitifera) var Thompson seedless, different treatments including compost from grape pomace, humic extract, microbial inoculant, and chemical NPK fertilization, were evaluated in a pot experiment using an Inceptisol soil. Organic materials were applied in four $\mathrm{C}$ rates: compost, $0,125,250$ y $500 \mathrm{~g} \mathrm{C}_{\text {pot }}^{-1}$; humic extract, $0,25,50$ and $100 \mathrm{~g} \mathrm{C}^{-1}{ }^{-1}$; compost and humic extract at their maximum $\mathrm{C}$ rates were also evaluated in absence of chemical fertilization, and microbial inoculant as bio-stimulant. Results showed a stronger root development in plants with compost and inoculant application. Furthermore, organic matter mineralization increased nutrient availability; this was evidenced by an increment of enzymatic activities, particularly $\beta$-glucosidase, acid phosphatase, and alkaline phosphatase, in all treatments receiving compost. On the other hand, the application of humic extract produced a larger increment in WSC per carbon unit applied compared to compost, meaning that the former would be more efficient than the latter to provide $\mathrm{C}$ for soil microorganisms. This study showed that the joint application of compost, mineral fertilizer, and microbial inoculant should be considered to improve root development in Table grape (Vitis vinifera L.) and soil quality, under Integrated Nutrient Management (INM) programs.
\end{abstract}

Keywords: Compost, humic substances, root density, enzymatic activity, soil quality indicators

\section{Introduction}

The root system plays an important role on physiological and biochemical development of plants due to its influence on nutrient and water uptake, exploratory capacity, and anchorage into soil (Páez- Garcia et al.,
2015). Therefore, a good root development is the key to achieve high yield and quality in table grape ( $\mathrm{Vi}$ tis vinifera L.) (Palma, 2006; Tomasi, 2016). Root growth pattern depends on age, variety, rootstock, 
nutritional status, climate, and environmental stresses (Callejas et al., 2009; Ruiz-Herrera et al., 2015). Smaller roots (2-6 $\mathrm{mm}$ in diameter) can emerge and grow both horizontally or vertically. The smaller roots are extended in a few extensions, which are generally thin (1-2 $\mathrm{mm}$ in diameter) with fast growth, but they die some weeks after emergence, and are replaced continuously (Terence et al., 2002). The root distribution of table grape in soils is influenced by diverse factors as temperature, texture, water, nutrient availability, $\mathrm{pH}$, organic matter content and tillage (Palma, 2006; Tomasi, 2016).

Table grapes are cultivated in arid and semi-arid regions under irrigated soils, where many times the plants are exposed to salinity and water stress. Orchards are commonly fertilized with NPK and micronutrients sources (Sierra, 2001). Nevertheless, soil agronomic management has been focused on organic matter to improve soil organic carbon (SOC), chemical-physical conditions, and water content in the root zone (Fincheira-Robles et al., 2016; Gaiotti et al., 2016). Commercial products based on humic and fulvic acids are available. The chemical extraction of humic and fulvic acids lead to the formation of three fractions: humic acids, fulvic acids and humins (Ortega and Fernández, 2007). The humic substances influence soil fertility through the improvement of soil structure, the increase chelating minerals and its degradation (Anderson and Schoenau, 1993; Scotti et al., 2015; Nardi et al., 2016). Furthermore, compost has been reported for its ability to reduce soil erosion and improve soil fertility and structure. Compost could improve structure, porosity, plant nutrients, enzymatic activities and the diversity of fungal and bacterial species with ability to stimulate plant growth (Martínez et al., 2010). Recently, Martínez et al. (2016) reported that compost quality depends heavily on chemical composition and sanitary parameters, where hydrolytic enzyme activities, total bacteria count and humic acid: fulvic acid ratios were the parameters of greater sensitivity. Additionally, microbial inoculants represent an alternative to formulate bioproducts containing living microorganisms or their metabolites in organic or inorganic substrates to promote plant growth (Vejan et al., 2016). The microbial pathways promote growth through the following mechanisms: nitrogen fixation, phosphate solubilization, iron uptake, phytohormones level modulation, excretion of antibiotic, enzymes and siderophores (Martínez-Viveros et al., 2010).

The application of carbon, as compost or humic extracts, and microbial inoculant can affect soil quality by improving physical, chemical, biochemical and microbiological soil properties, stimulating root development and plant. The objectives of this research were: 1) to determine the effect of two organic matter sources and a microbial inoculant on selected chemical and biological soil properties, and root and shoot development in table grape, variety Thompson seedless at establishment and 2) to relate soil properties and plant growth to determine the most sensitive soil quality indices explaining root and shoot development.

\section{Materials and Methods}

\subsection{Experimental design and plant material}

A pot experiment, under field conditions, was con-

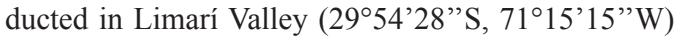
located in Coquimbo Region (Chile). This zone is one of the important table grape producing areas in Chile, with a Mediterranean climate and annual average rainfall of $100 \mathrm{~mm}$. The plant material utilized was Table grape Vitis vinifera L. var Thompson seedless (one-year-old) cultivated on their own roots, and planted on $20 \mathrm{~L}$ pots using an Inceptisol soil. Eighteen treatments and 6 replications were used and were dis- 
tributed in a complete randomized design (Table 1). Evaluated rates of compost were: 500, 1000 and 2000 $\mathrm{g}$ of product pot ${ }^{-1}$ equivalent to 125,250 , and $500 \mathrm{~g} \mathrm{C}$ pot $^{-1}$, respectively, while rates of humic extract were: 1000, 2000 and $4000 \mathrm{~mL}^{-1}$ pot $^{-1}$ equivalent to 25,50 and $100 \mathrm{~g} \mathrm{C} \mathrm{pot}^{-1}$, respectively. Both $\mathrm{C}$ sources were evaluated in the presence or absence of microbial inoculant. Furthermore, the maximum rates of amendments were evaluated with and without of mineral fertilizer application. Two controls were used: chemical fertilization and an absolute control without any application. Compost was added once at the beginning of the experiment and humic extract was applied in ten split applications during the season. Furthermore, microbial inoculant was added twice, while a fertilizer solution was applied in six equal splits during the season. The cultural practices, including irrigation and manual weed control, were the same for all treatments. The evaluation period corresponded to one growing season (September to May).

Table 1. Applied treatments

\begin{tabular}{|c|c|c|c|c|c|c|}
\hline Treatment & C source & $\begin{array}{c}\text { Chemical } \\
\text { fertilization }^{1}\end{array}$ & $\begin{array}{l}\text { Compost } \\
\left(\text { g pot }^{-1}\right)^{2}\end{array}$ & $\begin{array}{c}\text { Humic Extract } \\
\left(\mathrm{L} \text { pot }^{-1}\right)^{3}\end{array}$ & $\begin{array}{l}\text { Microbial inoculant } \\
\quad\left(\mathrm{mL} \mathrm{pot}^{-1}\right)^{4}\end{array}$ & $\begin{array}{l}\text { C rate } \\
\left(\text { g pot }^{1}\right)\end{array}$ \\
\hline $\mathbf{T}_{1}$ & Compost & Yes & 500 & & & 125 \\
\hline $\mathbf{T}_{3}$ & Compost & Yes & 2000 & & & 500 \\
\hline $\mathbf{T}_{4}$ & Compost & No & 2000 & & & 500 \\
\hline $\mathbf{T}_{7}$ & Compost & Yes & 2000 & & 400 & 500 \\
\hline $\mathbf{T}_{8}$ & Compost & No & 2000 & & 400 & 500 \\
\hline $\mathbf{T}_{9}$ & Humic extract & Yes & & 10 & & 25 \\
\hline $\mathbf{T}_{10}$ & Humic extract & Yes & & 20 & & 50 \\
\hline $\mathbf{T}_{11}$ & Humic extract & Yes & & 40 & & 100 \\
\hline $\mathbf{T}_{15}$ & Humic extract & Yes & & 40 & 400 & 100 \\
\hline $\mathbf{T}_{16}$ & Humic extract & No & & 40 & 400 & 100 \\
\hline $\mathbf{T}_{17}$ & None & Yes & & & & 0 \\
\hline $\mathbf{T}_{18}$ & None & No & & & & 0 \\
\hline
\end{tabular}

${ }^{1}$ Chemical fertilization: Novatec Solub $21 \circledR$, (stabilized ammonium with nitrification inhibitor 3,4 DMPP): $20 \mathrm{~g} \mathrm{pot}^{-1}$, phosphoric acid $15 \mathrm{~mL}_{\text {pot }}{ }^{-1}$ and potassium sulfate $10 \mathrm{~g} \mathrm{pot}^{-1} .{ }^{2}$ Application only at the beginning. ${ }^{3}$ Four applications $1,4,5,6 .{ }^{4}$ Two applications, one at $1^{\text {st }}$ week and other at week $6^{\text {th }}$.

\subsection{Amendment substrates and microbial inoculant}

Compost was obtained from an optimized co-composting process of grape pomace and goat manure and characterized according to the Chilean standard, NCh 2880/16 (INN, 2016). Humic extract was produced from the same compost, extracted during 18 hours using $0.3 \mathrm{M} \mathrm{KOH}$ and a ratio extractant: compost of 10:1. Humic extract was added in dilution 1:100 in water in order to prevent physicochemical instability and the damage to plant by EC (Ortega and Fernandez, 2007). Mineral fertilizer solution was made based on $25 \mathrm{~g} \mathrm{~N}_{\text {pot }}{ }^{-1}, 9 \mathrm{~g} \mathrm{P}_{2} \mathrm{O}_{5}$ pot $^{-1}$ and $30 \mathrm{~g} \mathrm{~K}_{2} \mathrm{O}$ pot ${ }^{-1}$, which are provided by Novatec Solub $21{ }^{\circledR}, \mathrm{H}_{3} \mathrm{PO}_{4}$ and $\mathrm{K}_{2} \mathrm{SO}_{4}$ 
$\left(50 \% \mathrm{~K}_{2} \mathrm{O}\right)$, respectively. The microbial inoculant consisted of a mixture of enzyme-producing rhizobacteria that included: Pseudomonas sp., Ochrobactrum anthropi, Brevundimonas sp., and Sphingomonas paucimobilis. The bacterial strains were isolated from compost derived from grape pomace and goat manure, with bio-stimulant action and formulated in a concentration of $6.2 \times 10^{10} \mathrm{CFU} \mathrm{mL}{ }^{-1}$. Microbial inoculant solution was applied at the rate of $200 \mathrm{~mL} \mathrm{pot}^{-1}$, twice during the season, in the corresponding treatments (see Table 1).

\subsection{Soil sampling}

Soil samples from each pot were collected at $20 \mathrm{~cm}$ depth at the end of the experiment. The samples were transported immediately to the laboratory to determine chemical, biochemical and microbiological properties.

\subsection{Measured variables}

Initially, the organic amendments were characterized according to the variables established by Chilean Standard NCh 2880-2016 (INN, 2016) and those proposed by Test Methods for Examination of Compost and Composting (Thompson et al., 2001). Soil samples were analyzed according to the methods of Sadzawka et al. (2004). Humic and fulvic acids contents $(\mathrm{HA}+\mathrm{FA})$ were determined by Anderson and Schoenau (1993), modified for compost.

Microbial populations were determined by the plate count method for isolating heterotrophic bacteria (nutritive agar), fungi (PDA agar), yeasts (YPG agar), actinomycetes (oat agar) and phosphate solubilizer microorganisms (PSB) (SMRS1 agar) (Martínez and Gutiérrez, 2010). The enzymatic activities were determined following the methods recommended by Dick et al. (1996), including $\beta$-glucosidase potential activity ( $p$-nitrophenol method) and acid and alkaline phosphatase potential activities ( $p$-nitrophenol method); urease activity was determined by Kandeler et al. (1999), using the indophenol blue technique (García et al., 2003).

\subsection{Evaluation of agronomical variables}

Trunk diameter (TD) and plant height were measurement after 0,8 , and 13 months from plantation. The diameter was measured with a caliper and the shoot cross sectional area (SCSA) was calculated as plant vigor indicator. In addition, at the final evaluation time, roots of each treatment were collected to determine root dry matter (DM) by washing the soil from each pot and oven desiccation at $70{ }^{\circ} \mathrm{C}$, until constant weight. Results were expressed as density units dividing the root dry mass by the volume of soil.

\subsection{Statistical analysis}

Data were analyzed using SAS statistical software (SAS, 2008). Data evaluation included analysis of variance (ANOVA) with protected LSD test $(p<0.05)$, and correlation analysis.

\section{Results}

\subsection{Quality of the organic amendments and base soil substrate}

The compost used for the experiment showed physical, chemical, biochemical, and toxicological characteristics that allowed its classification as mature compost (Martínez et al., 2016) type A (INN, 2016). On the other hand, humic extract presented lower $\mathrm{C}$ content, but similar $\mathrm{pH}$ and $\mathrm{EC}$ values compared with commercial humic extracts from leonardite (Ortega and Fernandez, 2007). The base soil showed low organic matter content, a property typically associated with Inceptisols soils present in semi-arid regions (Table 2). 
Table 2. Chemical and biochemical characteristics of soil, compost and humic extract used for the experiment.

\begin{tabular}{|c|c|c|c|c|}
\hline Variable & Unit & Base Soil & Compost & Humic extract \\
\hline Acid Phosphatase (UP) & $\mu \mathrm{g}$ p-nitrophenol $\mathrm{g}^{-1} \mathrm{~h}^{-1}$ & 203 & 849 & 3 \\
\hline Alkaline Phosphatase (UP) & $\mu \mathrm{g}$ p-nitrophenol $\mathrm{g}^{-1} \mathrm{~h}^{-1}$ & 185 & 1338 & 33 \\
\hline B-glucosidase (UBG) & $\mu \mathrm{g}$ p-nitrophenol $\mathrm{g}^{-1} \mathrm{~h}^{-1}$ & 78 & 238 & ND \\
\hline Urease (UU) & 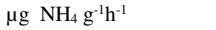 & 84 & 221 & 14 \\
\hline Humic acids & $\%$ & 0.54 & 5 & 2,34 \\
\hline $\mathrm{pH}$ & & 8.1 & 8.6 & 12.2 \\
\hline $\mathrm{EC}$ & $\mathrm{dS} \mathrm{m}^{-1}$ & 0.7 & 0.88 & 29.6 \\
\hline $\mathrm{N}-\mathrm{NH}_{4}$ & $\mathrm{mg} \mathrm{kg}^{-1}$ & 8.0 & 72 & 49 \\
\hline $\mathrm{N}-\mathrm{NO}_{3}$ & $\mathrm{mg} \mathrm{kg}^{-1}$ & 5.0 & 309 & 43 \\
\hline P- Olsen & $\mathrm{mg} \mathrm{kg}^{-1}$ & 7.0 & 55.6 & - \\
\hline Organic Matter & $\%$ & 1.4 & 44.5 & 0.80 \\
\hline Organic $\mathrm{C}$ & $\%$ & 1.5 & 24.7 & 0.25 \\
\hline Total N & $\%$ & 0.1 & 1.8 & 0.04 \\
\hline $\mathrm{C} / \mathrm{N}$ ratio & & 11.2 & 13.4 & 6.25 \\
\hline
\end{tabular}

\subsection{Effect of organic amendments application on soil properties}

Chemical properties, $\mathrm{pH}$ and EC, showed significant differences among treatments $(p<0.05)$. As expected, treatments with humic extract had higher $\mathrm{pH}$ and $\mathrm{EC}$ values compared with compost. However, at the end of the experiment the maximum EC values were slightly lower than the control with no organic amendments. The observed increase of $\mathrm{EC}$ values at the beginning of the experiment due to humic extract application triggered a significant reduction on microbial activity and plant growth, which could not be recovered during the season. Therefore, soil had to be leached to reduce EC. For this reason, only chemical properties were evaluated in treatments containing humic extract. Soil content of humic and fulvic acids was higher in all treatments receiving organic matter in comparison to the control, particularly when the maximum level of $\mathrm{C}$ was added $(p<0.05)$. The $\mathrm{NO}_{3}-\mathrm{N}$ levels were higher in compost treatments $(p<0.05)$ (Table 3).

Table 3. Effect of the applied treatments on selected soil chemical properties

\begin{tabular}{|c|c|c|c|c|c|c|c|c|c|}
\hline & Olsen-P & $\mathrm{N}-\mathrm{NH}_{4}$ & $\mathrm{~N}-\mathrm{NO}_{3}$ & Avail. $\mathrm{N}$ & $\mathrm{HFa}$ & OM & WSC & $\mathrm{pH}$ & $\mathrm{EC}$ \\
\hline & \multicolumn{4}{|c|}{ - } & \multicolumn{2}{|c|}{ 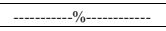 } & $\mathrm{mg} \mathrm{kg}^{-1}$ & & $\mathrm{dS} / \mathrm{m}$ \\
\hline $\mathrm{T}_{1}$ & $24.99 \pm 4.99$ & $4.33 \pm 4.08$ & $2.43 \pm 0.37$ & $6.76 \pm 4.15$ & $0.28 \pm 0.1$ & $0.69 \pm 0.08$ & $15.9 \pm 1.2$ & $7.02 \pm 0.7$ & $0.16 \pm 0.13$ \\
\hline $\mathrm{T}_{2}$ & $33.02 \pm 13.22$ & $2.47 \pm 1.34$ & $3.06 \pm 1.09$ & $5.54 \pm 2.35$ & $0.65 \pm 0.22$ & $1.6 \pm 0.38$ & $21.5 \pm 6.69$ & $7.28 \pm 0.06$ & $0.13 \pm 0.039$ \\
\hline $\mathrm{T}_{3}$ & $22.59 \pm 2.43$ & $5.17 \pm 0.8$ & $2.2 \pm 1.64$ & $7.37 \pm 0.88$ & $1.31 \pm 0.48$ & $2.61 \pm 0.75$ & $25.0 \pm 6.25$ & $7.23 \pm 0.42$ & $0.12 \pm 0.039$ \\
\hline $\mathrm{T}_{4}$ & $25.66 \pm 4.78$ & $1.69 \pm 0.84$ & $2.16 \pm 1.25$ & $3.86 \pm 0.9$ & $1.65 \pm 0.38$ & $3.04 \pm 1.25$ & $22.2 \pm 4.3$ & $7.86 \pm 0.83$ & $0.17 \pm 0.04$ \\
\hline$T_{5}$ & $24.16 \pm 9.65$ & $9.08 \pm 9.57$ & $3.16 \pm 2.26$ & $12.25 \pm 10.96$ & $0.43 \pm 0.12$ & $0.93 \pm 0.22$ & $15.9 \pm 1.2$ & $7.20 \pm 0.35$ & $0.22 \pm 0.12$ \\
\hline$T_{6}$ & $28.19 \pm 11.4$ & $8.88 \pm 10.7$ & $3.33 \pm 1.25$ & $12.21 \pm 10.96$ & $0.99 \pm 0.29$ & $1.47 \pm 0.74$ & $18.7 \pm 2.08$ & $6.95 \pm 0.16$ & $0.21 \pm 0.1$ \\
\hline $\mathrm{T}_{7}$ & $26.54 \pm 10.6$ & $2.9 \pm 0.78$ & $3.1 \pm 0.81$ & $6.01 \pm 1.6$ & $1.61 \pm 0.33$ & $2.49 \pm 0.52$ & $27.08 \pm 4.17$ & $7.02 \pm 0.27$ & $0.14 \pm 0.046$ \\
\hline $\mathrm{T}_{8}$ & $18.79 \pm 8.32$ & $6.71 \pm 5.6$ & $3.06 \pm 1.68$ & $9.77 \pm 7.02$ & $2.29 \pm 0.90$ & $3.20 \pm 0.51$ & $28.5 \pm 5.24$ & $7.21 \pm 0.35$ & $0.16 \pm 0.06$ \\
\hline $\mathrm{T}_{9}$ & $15.36 \pm 5.83$ & $1.09 \pm 1.04$ & $1.06 \pm 0.46$ & $2.16 \pm 0.99$ & $0.33 \pm 0.06$ & $0.52 \pm 0.06$ & $12.5 \pm 2.08$ & $8.18 \pm 0.13$ & $0.18 \pm 0.04$ \\
\hline$T_{10}$ & $16.95 \pm 7.47$ & $2.68 \pm 1.6$ & $1.56 \pm 0.46$ & $4.24 \pm 2.01$ & $0.42 \pm 0.05$ & $0.60 \pm 0.1$ & $14.5 \pm 5.58$ & $8.63 \pm 0.43$ & $0.22 \pm 0.1$ \\
\hline$T_{11}$ & $24.3 \pm 22.6$ & $0.63 \pm 0.58$ & $1.9 \pm 1.17$ & $2.56 \pm 1.66$ & $0.40 \pm 0.13$ & $0.60 \pm 0.06$ & $16.67 \pm 2.08$ & $8.81 \pm 0.47$ & $0.31 \pm 0.13$ \\
\hline$T_{12}$ & $12.0 \pm 7.0$ & $1.16 \pm 1.49$ & $1.83 \pm 0.61$ & $2.99 \pm 1.50$ & $0.44 \pm 0.12$ & $0.61 \pm 0.05$ & $15.97 \pm 5.21$ & $8.9 \pm 0.08$ & $0.23 \pm 0.01$ \\
\hline$T_{13}$ & $14.6 \pm 7.0$ & $2.8 \pm 2.91$ & $1.13 \pm 0.5$ & .07 & $0.36 \pm 0.11$ & $0.66 \pm 0.15$ & $12.5 \pm 2.08$ & $8.09 \pm 0.01$ & $0.22 \pm 0.02$ \\
\hline$T_{14}$ & $24.2 \pm 19.6$ & $1.12 \pm 0.74$ & $0.93 \pm 0.32$ & $2.05 \pm 0.97$ & $0.35 \pm 0.07$ & $0.64 \pm 0.17$ & $11.1 \pm 5.24$ & $8.4 \pm 0.11$ & $0.18 \pm 0.01$ \\
\hline$T_{15}$ & $26.11 \pm 2.23$ & $2.71 \pm 1.53$ & $1.46 \pm 0.43$ & $4.19 \pm 1.66$ & $0.41 \pm 0.17$ & $0.5 \pm 0.04$ & $18.06 \pm 4.34$ & $8.94 \pm 0.54$ & $0.34 \pm 0.2$ \\
\hline$T_{16}$ & $11.83 \pm 5.77$ & $1.55 \pm 1.26$ & $0.6 \pm 0.33$ & $2.15 \pm 0.91$ & $0.43 \pm 0.19$ & $0.66 \pm 0.17$ & $17.36 \pm 3.18$ & $9.01 \pm 0.47$ & $0.52 \pm 0.02$ \\
\hline$T_{17}$ & $11.12 \pm 2.68$ & $3.23 \pm 1.57$ & $0.66 \pm 0.35$ & $3.89 \pm 1.81$ & $0.23 \pm 0.17$ & $0.67 \pm 0.1$ & $13.19 \pm 3.42$ & $7.45 \pm 0.52$ & $0.17 \pm 0.08$ \\
\hline$T_{18}$ & $15.7 \pm 2.7$ & $1.72 \pm 1.43$ & $1.23 \pm 0.85$ & $2.95 \pm 2.28$ & $0.35 \pm 0.13$ & $0.56 \pm 0.12$ & $12.5 \pm 2.8$ & $7.58 \pm 0.56$ & $0.17 \pm 0.09$ \\
\hline$\overline{\mathrm{LSD}^{2}{ }_{\text {TR }}}$ & $\mathrm{n}$ & $\mathrm{n}$ & & 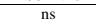 & & 0.74 & & 0.91 & 0.19 \\
\hline $\mathrm{LSD}_{-}^{3}$ & 5.80 & 2.76 & 0.59 & 2.58 & 0.31 & 0.44 & 2.95 & 0.32 & 0.076 \\
\hline $\mathrm{LSD}^{4}{ }_{-1 \mathrm{~N}}$ & $\mathrm{~ns}$ & $\mathrm{~ns}$ & ns & $\mathrm{ns}$ & ns & ns & ns & ns & $\mathrm{ns}$ \\
\hline
\end{tabular}

${ }^{1}$ Variation is expressed as standard deviation. T: Treatment; HFa: humic and fulvic acids; OM: organic matter; WSC: water-soluble carbon; EC: electrical conductivity. ${ }^{2}$ Least significant difference; ${ }^{3}$ Compares compost vs humic extract; ${ }^{4}$ compares inoculated vs non inoculated ns: not significant $(p>0.05)$ 
Soil organic matter (SOM) showed an increase with $\mathrm{C}$ rate in treatments with compost. In contrast, humic extract did not produce any change on total SOM (Figure 1a,b). However, the water soluble carbon (WSC) increased in both organic amendments according to $\mathrm{C}$ rate (Figure 2). Interestingly, the

A

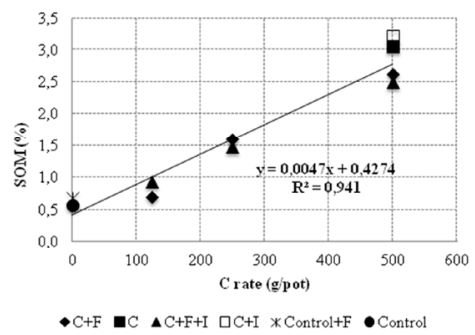

slope of WSC increase with $\mathrm{C}$ rate was larger for humic extract $\left(0.048 \mathrm{mg} \mathrm{kg}^{-1} \mathrm{~g} \mathrm{C}^{-1}\right.$, Figure $\left.2 \mathrm{~b}\right)$ than compost $\left(0.0257 \mathrm{mg} \mathrm{kg}^{-1} \mathrm{~g} \mathrm{C}^{-1}\right.$, Figure 2a). Thus, humic extract would be more efficient than compost to provide $\mathrm{C}$ for soil microorganisms, particularly soluble $\mathrm{C}$.

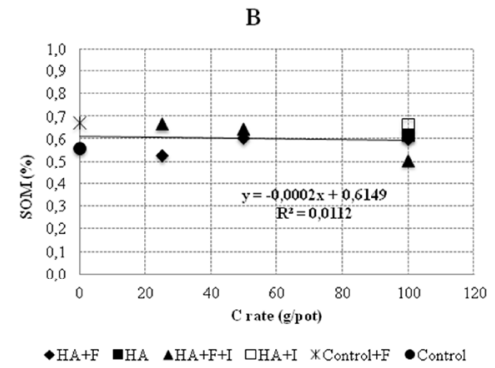

Figure 1. Variation of Soil Organic Matter (SOM) as function of $\mathrm{C}$ rate applied as: A) Compost and B) Humic extract.

A

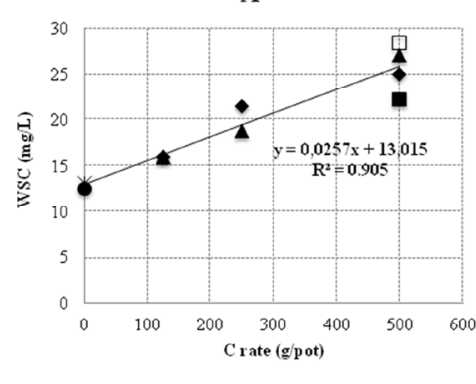

$\bullet \mathrm{C}+\mathrm{F} \backsim \mathrm{C} \Delta \mathrm{C}+\mathrm{F}+\mathrm{I} \quad \square \mathrm{C}+\mathrm{I} *$ Control $+\mathrm{F} \bullet$ Control
B

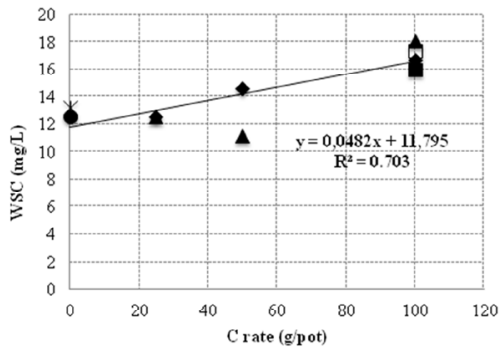

$\bullet \mathrm{HA}+\mathrm{F} \square \mathrm{HA} \triangle \mathrm{HA}+\mathrm{F}+\mathrm{I} \square \mathrm{HA}+\mathrm{I} *$ Control+F $\bullet$ Control

Figure 2. Variation of Water Soluble Carbon (WSC) as function of C rate applied as: A) Compost and B) Humic extract.

\subsubsection{Effect of Compost application on Biochemical Properties}

Biochemical analysis revealed that all compost treatments increased potential activity of $\beta$-glucosidase and acid phosphatase enzymes, compared to the control $(p<0.05)$. On the other hand, high rates of $\mathrm{C}$ provided by compost and humic extract decreased urease potential activity $(p<0.05)$. Finally, the presence of microbial inoculant increased $\beta$-glucosidase potential activity while urease one was decreased (Table 4). 
Table 4. Effect of compost applications on soil enzymatic activities ${ }^{1}$

\begin{tabular}{|c|c|c|c|c|}
\hline \multirow[t]{2}{*}{$\mathrm{T}$} & $\begin{array}{c}\beta \text {-Glucosidase } \\
\text { (UBG) }\end{array}$ & $\begin{array}{c}\text { Acid phosphatase } \\
\text { (AcUP) }\end{array}$ & $\begin{array}{l}\text { Alkaline phosphatase } \\
\text { (alkUP) }\end{array}$ & \multirow{2}{*}{$\begin{array}{c}\begin{array}{c}\text { Urease } \\
(\mathrm{UU})\end{array} \\
\mu \mathrm{g} \mathrm{NH} \mathrm{NH}_{4} \mathrm{~g}^{-1}\end{array}$} \\
\hline & \multicolumn{3}{|c|}{ - } & \\
\hline $\mathrm{T}_{1}$ & $45.8 \pm 22.12$ & $117.9 \pm 34.95$ & $184.3 \pm 95.35$ & $119.3 \pm 40.03$ \\
\hline $\mathrm{T}_{2}$ & $49.3 \pm 11.17$ & $164.1 \pm 31.52$ & $264.5 \pm 70.19$ & $222.7 \pm 45.87$ \\
\hline $\mathrm{T}_{3}$ & $69.8 \pm 42.4$ & $269.5 \pm 44.03$ & $372.6 \pm 151.76$ & $230.7 \pm 103.04$ \\
\hline $\mathrm{T}_{4}$ & $49.6 \pm 38.96$ & $329.0 \pm 167.19$ & $634.2 \pm 526.56$ & $201.5 \pm 33.12$ \\
\hline $\mathrm{T}_{5}$ & $50.2 \pm 16.60$ & $117.1 \pm 8.43$ & $304.0 \pm 163.48$ & $146.5 \pm 57.18$ \\
\hline $\mathrm{T}_{6}$ & $49.6 \pm 2.72$ & $150.8 \pm 73.48$ & $206.7 \pm 93.25$ & $168.8 \pm 44.45$ \\
\hline $\mathrm{T}_{7}$ & $65.2 \pm 5.94$ & $251.4 \pm 47.36$ & $366.0 \pm 75.54$ & $151.1 \pm 8.06$ \\
\hline $\mathrm{T}_{8}$ & $69.6 \pm 19.75$ & $369.0 \pm 149.94$ & $415.7 \pm 208.12$ & $165.7 \pm 56.36$ \\
\hline $\mathrm{T}_{17}$ & $26.74 \pm 8.02$ & $138.7 \pm 47.6$ & $219.2 \pm 51.16$ & $258.3 \pm 38.64$ \\
\hline $\mathrm{T}_{18}$ & $47.5 \pm 9.08$ & $158.7 \pm 26.16$ & $205.2 \pm 17.06$ & $229.6 \pm 13.59$ \\
\hline $\mathrm{LSD}_{-\mathrm{TR}}$ & 36.69 & 185.29 & ns & ns \\
\hline $\mathrm{LSD}_{\text {-OM }}^{3}$ & 18.91 & 112.9 & ns & $\mathrm{ns}$ \\
\hline $\mathrm{LSD}^{4}{ }_{-\mathrm{IN}}$ & 20.54 & ns & $\mathrm{ns}$ & 34.50 \\
\hline
\end{tabular}

$\mathrm{T}$ : Treatment

${ }^{1} \mathrm{UBG}$ and $\mathrm{UP}=\mu \mathrm{g} p$-nitrophenol $\mathrm{g}^{-1} \mathrm{~h}^{-1} ; \mathrm{UU}=\mu \mathrm{g} \mathrm{NH} \mathrm{g}^{-1} \mathrm{~h}^{-1}$. Variation is expressed as standard deviation

${ }^{2}$ Least significant difference; ${ }^{3}$ Compares compost vs control; ${ }^{4}$ compares inoculated vs non inoculated

3.2.2. Effect of Compost application on Microbial Populations

Microbiological analysis revealed no significant effect of treatments on the evaluated microbial populations, except on yeasts, where compost at minimum $\mathrm{C}$ level had the highest yeast concentration $(\mathrm{p}<0.0001)$. On the other hand, the presence of inoculant tended to increase fungi populations (Table 5).

Table 5. Effect of compost applications on microbial soil populations

\begin{tabular}{|c|c|c|c|c|}
\hline \multirow{2}{*}{$\mathrm{T}$} & PSB & Actinomycetes & Fungi & Yeasts \\
\hline & \multicolumn{4}{|c|}{ 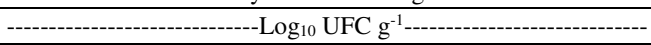 } \\
\hline $\mathrm{T}_{1}$ & $6.4 \pm 0.3$ & $6.0 \pm 0.2$ & $6.3 \pm 0.3$ & $6.5 \pm 0.3$ \\
\hline $\mathrm{T}_{2}$ & $6.2 \pm 0.5$ & $6.0 \pm 0.2$ & $6.2 \pm 0.3$ & $6.5 \pm 0.4$ \\
\hline $\mathrm{T}_{3}$ & $6.3 \pm 0.2$ & $6.2 \pm 0.2$ & $6.2 \pm 0.3$ & $6.2 \pm 0.5$ \\
\hline $\mathrm{T}_{4}$ & $5.2 \pm 1.4$ & $5.7 \pm 0.6$ & $5.8 \pm 0.8$ & $7.4 \pm 0.3$ \\
\hline $\mathrm{T}_{5}$ & $6.4 \pm 0.3$ & $5.8 \pm 0.4$ & $6.7 \pm 0.6$ & $6.4 \pm 0.6$ \\
\hline $\mathrm{T}_{6}$ & $6.2 \pm 0.3$ & $6.6 \pm 0.3$ & $6.3 \pm 0.2$ & $6.7 \pm 0.4$ \\
\hline $\mathrm{T}_{7}$ & $6.7 \pm 0.2$ & $6.2 \pm 0.3$ & $6.6 \pm 0.1$ & $6.1 \pm 0.3$ \\
\hline $\mathrm{T}_{8}$ & $6.4 \pm 0.2$ & $6.6 \pm 0.3$ & $6.5 \pm 0.4$ & $5.8 \pm 0.2$ \\
\hline $\mathrm{T}_{17}$ & $6.3 \pm 0.0$ & $6.1 \pm 0.1$ & $6.3 \pm 0.3$ & $6.0 \pm 0.3$ \\
\hline $\mathrm{T}_{18}$ & $6.4 \pm 0.4$ & $5.9 \pm 0.4$ & $6.7 \pm 0.2$ & $6.5 \pm 0.4$ \\
\hline $\mathrm{LSD}^{2}{ }_{\mathrm{TR}}$ & ns & 0.53 & $\mathrm{~ns}$ & 0.65 \\
\hline $\mathrm{LSD}_{\text {_OM }}^{3}$ & ns & $\mathrm{ns}$ & ns & 0.54 \\
\hline $\mathrm{LSD}^{4} \_\mathrm{IN}$ & ns & $\mathrm{ns}$ & 0.31 & $\mathrm{~ns}$ \\
\hline
\end{tabular}

T: Treatment

${ }^{1}$ PSB: phosphate solubilizer bacteria. Variation is expressed as standard deviation.

${ }^{2}$ Least significant difference; ${ }^{3}$ Compares compost vs control; ${ }^{4}$ compares inoculated vs non inoculated 
3.2.3. Effect of compost application on agronomic variables: Effect on root development

Results indicated that the treatment including compost, microbial inoculant and chemical fertilizers $\left(\mathrm{T}_{5}\right)$ improved root growth development compared

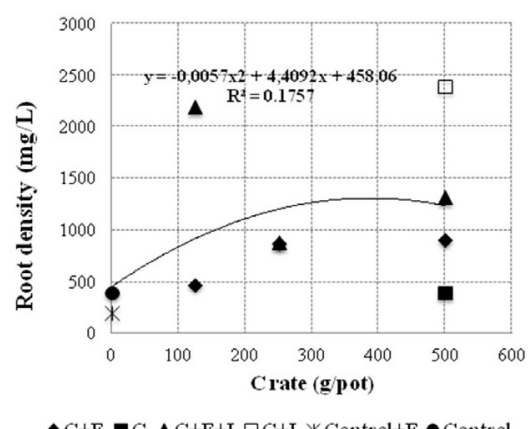

$\bullet \mathrm{C}+\mathrm{F} \square \mathrm{C} \Delta \mathrm{C}+\mathrm{F}+\mathrm{I} \square \mathrm{C}+\mathrm{I} * \mathrm{Control}+\mathrm{F} \bullet$ Control to the other treatments. Root density showed a clear tendency to increase with compost rate. Additionally, the application of microbial inoculant potentiated this effect as shown in treatments that included it (Figure 3). Similar results were observed for shoot cross sectional area (data not shown).

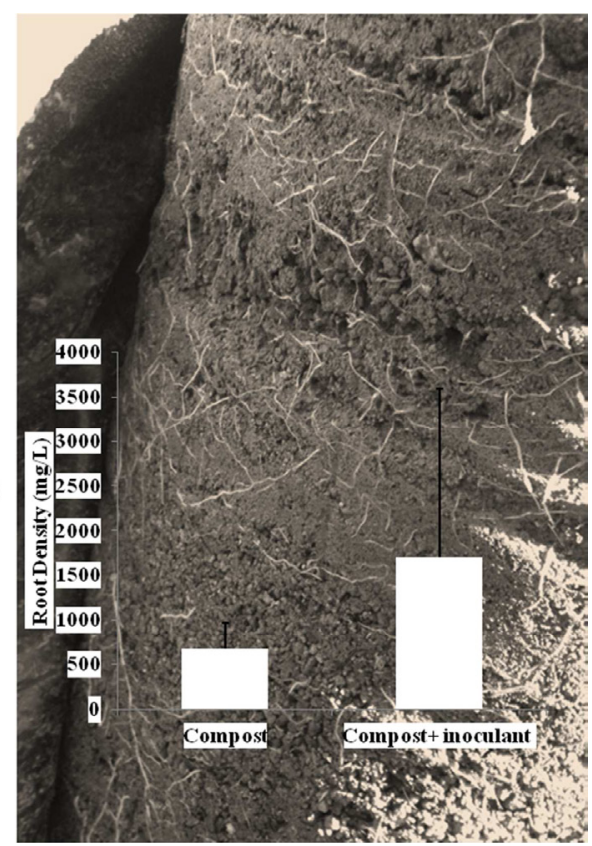

Figure 3. Plant root density as a function of $\mathrm{C}$ rate from compost (C) in presence or absence of chemical fertilization (F) and inoculant (I)

The correlation analysis (data not shown) performed indicated that the effect of humic extract on $\mathrm{pH}$ and $\mathrm{EC}$, root dry mass, enzymatic activity and microbial population were negatively correlated with these variables. Furthermore, a direct association between root density and available $\mathrm{N}$, acid phosphatase, and $\beta$-glucosidase was found. It was evidenced that humic and fulvic acids, organic matter and WSC content where positively correlated with phosphatases and $\beta$-glucosidase potential activities, and soil yeast concentration.

\section{Discussion}

Vitis vinifera $\mathrm{cv}$ Thompson seedless is a fruit species cultivated in a wide range of soil types and climatic conditions. Normally, table grape requires the application of nitrogen, phosphorus, potassium, and some micronutrients such as iron, boron, manganese and zinc to reach high production levels and fruit quality (Palma, 2006; Tomasi, 2016). Nitrogen is one of the most important and limiting nutrients in table grape, particularly in spring season. Mineral $\mathrm{N}$ fertilizers 
should be applied when table grape can absorb and assimilate $\mathrm{N}$ to build or supplement reserves, minimizing losses through leaching and denitrification processes. The period between "bloom" and "veraison" has a high $\mathrm{N}$ requirement (Palma, 2006). Besides, the available $\mathrm{N}$ will allocate and incorporate into permanent vine structures for storage when shoot growth is slow. During this period vines may accumulate up to $50 \%$ of annual $\mathrm{N}$ requirement. In contrast, the active root growth and mineral uptake is generally minimal during the budbreak period, thus $\mathrm{N}$ demand is supplied primarily from reserves stored in the roots and other permanent woody structures.

In the present study, compost supplied an equivalent rate of $36 \mathrm{~kg}$ of total $\mathrm{N} \mathrm{ha}^{-1}$ out of which, we estimated a maximum $\mathrm{N}$ mineralized of $10 \mathrm{~kg} \mathrm{~N}$ $\mathrm{ha}^{-1}$ season $^{-1}$. This amount probably did not influence plant response as mineral fertilization. However, $\mathrm{N}$ requirements at establishment are low, reaching $30 \mathrm{~kg} \mathrm{~N} \mathrm{ha}^{-1}$. Flavel et al. (2005) indicated that grape marc compost applied in $0-10 \mathrm{~cm}$ depth in sandy soils of Australia increased 2.7\% total $\mathrm{N}$ with high mineralization rates produced during the first days (2.4 to $7.4 \mathrm{mg} \mathrm{N} \mathrm{kg}^{-1}$ day $^{-1}$ ) in response to the decomposition of the soluble fractions of compost. The $\mathrm{N}$ supply derived from compost and other organic amendments should be considered in fertilizer programs; approximately $30 \%$ of the total nitrogen provided by compost is available during the season.

The mineral fertilizer applied in this experiment, Novatec Solub 21 $1_{\text {TM }}$, contains stabilized ammonium by the addition of the nitrification inhibitor 3,4 DMPP, which improves $\mathrm{N}$ use efficiency, reducing $\mathrm{NO}_{3}-\mathrm{N}$ losses by leaching and denitrification, increasing $\mathrm{N}$ uptake (Molina and Ortega, 2006). Available $\mathrm{N}$ was larger in treatments including compost, which was probably associated to mineralization of organic $\mathrm{N}$ and/or lower $\mathrm{N}$ losses.
Additionally, humic and fulvic acids triggered positive effects on plants, stimulating root development by improved nutrient uptake, morphological changes, and larger chlorophyll leaf content, while in the soil have beneficial effects on $\mathrm{pH}$, structure, water-air retention capacity, microbial population and acts a buffer solution in cation exchange capacity (Trevisan et al., 2010). The present study showed an increase of $\mathrm{HFa}$ content with $\mathrm{C}$ rate, especially when compost was used, as a larger value was observed in the treatment composed by compost and microbial inoculant $\left(\mathrm{T}_{8}\right)$. These results can be explained by a larger microbial activity, based on the use of compost as substrate during mineralization of organic matter and HFa production. Additionally, a positive correlation was found between soil HFa content and both alkaline and acid phosphatases as well as with actinomycetes population, indicating the presence of hydrolytic bacteria. However, a negative correlation between $\mathrm{HFa}$ and dry root biomass was observed, probably since the application of humic extract increased $\mathrm{pH}$ and EC generating suppression on root growth. Humic extracts are associated with direct growth promotion effects related to hormone-like activity which can modified modify plant physiology (Nardi et al., 2016).

Other studies have shown that large amendment rates increase soil carbon. Albaladejo et al. (2008) observed a 2-fold increase in soil carbon concentration after the application of 260 ton $\mathrm{ha}^{-1}$ of uncomposted organic municipal solid waste. Furthermore, Morlat and Chausson (2008) showed that the accumulative rate of 256-320 ton ha $^{-1}$ of compost in vineyards during 16 year exhibited 2-fold increase on carbon concentration, respect to the control soil. In this experiment WSC concentration in soil improved with $\mathrm{C}$ rate, regardless of the $\mathrm{C}$ source; however, the rate of increase was larger for humic extract than for 
the compost, probably due to the content of WSC in humic extract.

Diverse studies have associated compost application with the increase of soil enzymatic activities due to mineralization processes and microorganism activity (Martínez et al., 2016; Ciaccia et al., 2017). In contrast, other reports have indicated that the activity of enzymes such as proteases, urease and desaminases have decreased due to toxic effects caused by trace elements present in organic amendments (Heargreaves et al., 2008). In the present study, a significant increase on $\beta$-glucosidase, acid and alkaline phosphatase potential activities was observed in compost treatments. Carbon sources as cellulose, lignin, starch, proteins, organic $\mathrm{P}$, and other nutrients present in compost, besides the presence of microorganisms, stimulated biological activity and the continue mineralization process (Martínez et al., 2016). In contrast, the humic extract contains $\mathrm{C}$ sources as polyphenolic compounds and very low microbial activity, due to its high $\mathrm{EC}$ and $\mathrm{pH}$. Besides, urease activity decreased probably by application of compost and mineral fertilizer that maintained $\mathrm{N}-\mathrm{NH}_{4}$ levels in soil.

Additionally, organic amendments enhance soil microbial diversity that includes functional groups as mycorrhizal fungi and beneficial rhizosphere bacteria (Heargreaves et al., 2008). Organic matter content is an important factor that influences microbial population, particularly by the presence of labile and organic sources of $\mathrm{C}$. The results obtained in this study suggest that $\mathrm{C}$ treatments had a significant effect on yeast population, indicating that, probably, high $\mathrm{C}$ rates depressed the population by competence for substrate and space between native terroir yeasts and microorganisms added through compost. Furthermore, humic extract could suppress the microbial populations due to its high EC.
In contrast, other reports have reported that low concentration of humus-like substances extracted from compost stimulate the microbial population and vegetative biomass production.

According to the above-mentioned results, compost could be an economic and ecologic alternative to improve soil characteristics to induce plant growth promotion (Heargreaves et al., 2008; Fincheira-Robles et al., 2016). Composted grape pomace constitutes an important option to increase soil OM content improving physical, chemical and biological properties to soil ecosystem (FincheiraRobles et al., 2016). Ferrer et al. (2001) reported a positive effect using 1-4 ton $\mathrm{ha}^{-1}$ of grape marc compost as a soil conditioner for corn seed germination in greenhouses, while Gaioti et al. (2016), showed that grape marc compost provided some benefit in returning nutrients into the vineyard. Moreover, Kim et al. (2015) reported that the mixture of compost, vermicompost and rice straw compost promoted the growth of root on soybean and sweet corn. In the present study, it was observed that compost generated an improvement on root synthesis and no negative effects were observed, suggesting an important role in promoting rhizogenesis, particularly in nursery vines. Probably, compost affects critical soil variables that induce root distribution of grapevines as texture, aeration, temperature, nutrient availability and organic matter content. Additionally, it was observed that compost in combination with mineral fertilizers and microbial inoculant, resulted in improved root density. Chemical fertilization provides elements required by the plant and microorganisms; compost generates an adequate environment in terms of physical and chemical conditions for availability of these nutrients, and the microbial inoculant provides beneficial microorganisms that mineralize organic matter, releasing more nutrients 
and synthesizing humic substances (Ortega, 2015). Regarding the role of humic extract in root development, Ortega and Fernandez (2007) reported an increase in shoot and root biomass with $\mathrm{C}$ rate applied as humic substances, which was extracted from vermicompost and leonardite. In the present study, the high EC of humic extract caused negative effects in root development, despite the dilution performed before its application.

\section{Conclusions}

The use of compost as organic amendment during establishment of table grape improved the biological soil quality, favored the increased of humic and fulvic acids content and had a positive effect on root promotion in comparison with humic extract treatments and controls. The application of compost, mineral fertilization and microbial inoculant, as INM, produced the largest root dry matter. The use of humic extract must be done controlling the EC to avoid negative effects on biological soil activity and plant root synthesis. Additionally, it was possible to establish that phosphatases and $\beta$-glucosidase potential activity, WSC and soil humic and fulvic acids contents, are sensitive variables, which are strongly correlated with root growth (root density) and would be adequate to evaluate soil quality in table grape agroecosystems.

\section{Acknowledgements}

This research was supported by FONDECYT 1130975 and USM Projects 28.09.49 and 28.11.69. The authors are grateful to Mr. Walter Cortes for his support with field work, and Dr. Monika Wimmer in Plant Physiology Lab. INRES- Uni Bonn, BonnGermany, and GISPA-group, USM, Chile for their valuable help.

\section{References}

Albaladejo, J., Lobez J., Boix-Fayos C., Barbera G., Martínez-Mena, M. 2008. Long-term effect of a single application of organic refuse on carbon sequestration and soil physical properties. J. Environ. Qual. 37(6), 2093-2099.

Anderson, D. W., Schoenau, J. J. 1993. Soil humus fractions. in: Carter, M. R. (Ed.). Soil sam- pling and methods of analysis. Canadian Society of Soil Science. Lewis Publisher. London, England, pp. 391-397.

Callejas, R., Canales, P., García de Cortázar, V. 2009. Relationship between root growth of Thompson seedless grapevines and soil temperature. Chilean J. Agric. Res. 69(4), 496-502.

Dick, R., Breakwell, D., Turco, R. 1996. Chapter $15^{\text {th }}$ Soil enzyme activities and biodiversity measurements as integrative microbiological indicators, In: Doran, J., Jones, A. (Eds.), Methods for Assessing Soil Quality. Special Publication 49. SSSA. Soil Science Society of America, USA, pp. 247-271.

Ciaccia, C., Ceglie, F., Tittarelli, F., Antichi, D., Carlesi, S., Testani, E., Canali, S. 2017. Green manure and compost effects on N-P dynamics in Mediterranean organic stockless systems. J. Soil Sci. Plant Nutr. 17 (3), 751-769.

Ferrer, J., Paez, G., Marmol, Z., Ramones, E., Chandler, C., Marin, M., Ferrer, A. 2001. Agronomic use of biotechnologically processed grape wastes. Bioresour. Technol. 76, 39-44.

Fincheira-Robles, P., Martínez-Salgado M., OrtegaBlu R., Janssens M. 2016. Compost and humic substance effects on soil parameters of Vitis vinifera L cv Thompson seedless Scientia Agropecuaria. 7 (3), $291-296$. 
Flavel, T.C., Murphy, D.V., Lalor, B., Fillery, I.R. 2005. Gross N mineralization rates after application of composted grape marc to soil Soil Biol. Biochem. 37(7), 1397-1400.

Gaiotti, F., Marcuzzo, P., Battista, F., Belfiore, N., Petoumenou, D., Tomasi, D. 2016. Compost amendment effects on grapevine root density and distribution. Acta Hortic. 1136, 115-120.

García, C., Gil, F., Fernández, T., Trasar, C. 2003. Técnicas de análisis de parámetros bioquímicos en suelos: Medida de actividades enzimáticas y biomasa microbiana. Ediciones Mundiprensa. Spain, pp. 123-148

Heargreaves, J., Adl, M., Warman, P. 2008. A review of the use of composted municipal solid waste in agriculture. Agric Ecosyst Environ. 123, 1-14.

Instituto Nacional de Normalización (INN). 2016. Compost- Quality requirements and classification 2880-2016. 16 p.

Kalaichelvi, K., Chinnusamyl, C., Swaminathan, A. 2006. Exploiting the natural resource - lignite humic acid in agriculture - a review. Agric. Rev. 27(4), $276-283$.

Kandeler, E., Stemmer, M., Klimanek, E.M. 1999. Response of soil microbial biomass, urease and xylanase within particle size fractions to long-term soil management. Soil Biol. Biochem. 31(2), 261-273.

Kim, M., Shim, C., Kim, Y., Hong, S., Park, J., Han, E., Kim, J., Kim, S. 2015. Effect of aerated compost tea on the growth promotion of lettuce, soybean, and sweet corn in organic cultivation. Plant Pathol. J. 31(3), 259-268.

Martínez, M., Gutiérrez, V. 2010. Bacterias Fosfato Solubilizadoras. In: Martínez M., Pedroza, A., Gutiérrez V. (Eds.). Manual de Técnicas en Microbiología Ambiental. Ed Universidad Santa Maria, Santiago, Chile, pp. 31-41.
Martínez, M., Gutiérrez, V., Novo, R. 2010. Microbiología aplicada al manejo sustentable de suelos y cultivos. Editorial USM. Santiago, Chile. 235p.

Martínez, M, Ortega, R, Janssens, M, Angulo, J., Fincheira, P. 2016. Selection of maturity indices for compost derived from grape pomace. J. Soil Sci. Plant Nutr. 16(2), 262-267.

Martínez-Viveros, O., Jorquera, M., Crowley, D., Gajardo, G., Mora, M. 2010. Mechanisms and practical considerations involved in plant growth promotion by rhizobacteria. J. Soil Sci. Plant Nutr. 10(3), 293-319.

Molina, M., Ortega, R. 2006. Evaluation of the nitrification inhibitor 3,4-dimethylpyrazole phosphate (DMPP) in two Chilean soils. J Plant Nutr. 29(3), 521-534.

Morlat, R., Chausson, R. 2008. Long-term additions of organic amendments in a Loire Vally vineyard I Effects of properties of a calcareous sandy soil. Am. J. Enol. Vitic. 59, 353-363.

Nardi, S., Pizzeghello, D., Schiavon, M., Eartani, A. 2016. Plant biostimulants: physiological responses induced by protein hydrolyzed-based products and humic substances in plant metabolism. Sci. Agric. 73(1), 18-23.

Ortega, R. 2015. Integrated nutrient management in conventional intensive horticulture production systems. Acta Hort. 1076,159-164.

Ortega, R., Fernández, M. 2007. Agronomic evaluation of humic extract derived from earthworm humic substances. J. Plant Nutr. 30(12), 2091-2104.

Páez-Garcia, A., Motes, C., Scheible, W., Chen, R., Blancaflor, E., Monteros, M. 2015. Root traits and phenotyping strategies for plant improvement. Plants. 4(2), 334-355.

Palma, J. 2006. Estrategia de fertilización en vid de mesa diseños y monitoreos. Guía de manejo nutrición vegetal de especialidad: Uva. Soquimich Nitratos S.A. Chile. 136 p. 
Ruiz-Herrera, L., Shane, M., López-Bucio, J. 2015. Nutritional regulation of root development. Wiley Interdiscip Rev Dev Biol. 4(4), 432-443.

Sadzawka, A., Grez, R., Mora, M.L., Saavedra, N., Carrasco, M.A., Rojas, C. 2004. Métodos de análisis recomendados para suelos chilenos, programa de normalización de técnicas y acreditación de laboratorios para los análisis de suelos y tejidos vegetales. pp 6-63.

Scotti, R., Bonanomi, G., Scelza, R., Zoina, A., Rao, M. 2015. Organic amendments as sustainable tool to recovery fertility in intensive agricultural systems. J. Soil Sci. Plant Nutr. 15(2), 333-352.

Sierra, C. 2001. Fertilización en vides de mesa. Boletín INIA $\mathrm{N}^{\circ}$ 74. Instituto de investigaciones Agropecuarias- INIA. La Serena. Chile. 56 p.

Terence, R.B., Dunst, R.M., Joy, P. 2002. Seasonal dry matter, starch, and nutrient distribution in "Concord" grapevine roots. HortScience. 37(2), 313-316.
Thompson, W., Leege, P., Millner, P., Watson, M. 2001. Test Methods for Examination of Composting and Compost (TMECC). The US Composting Council Research and Education Foundation and the United States Department of Agriculture. United States. TMECC.

Tomasi, D. 2016. Grape quality starts at the roots. Acta Hortic. 1136, 1-10.

Trevisan, S., Francioso, O., Quaggiotti, S., Nardi, S. 2010. Humic substances biological activity at the plant-soil interface from environmental aspects to molecular factors. Plant Signal Behav. 5(6), 635-643.

Vejan, P., Abdullah, R., Khadiran, T., Ismail, S., Nasrulhaq Boyce, A. 2016. Role of plant growth promoting rhizobacteria in agricultural sustainability-A Review. Molecules. 21(5), 573. 